\title{
Design and Implementation of Information Representation System for Intelligence Acquisition
}

\author{
Ru Liu, Menghui Li, Huina Zhang, Yurong Huang, Jingyan Zhou \\ Beijing Municipal Institute of Science and Technology Information, Beijing 100120, \\ china
}

\begin{abstract}
Keywords: Information Representation System; Visualization; Intelligence Acquisition; Hall for WorkShop of Metasynthetic Engineering
\end{abstract}

\begin{abstract}
Information Representation System (IRS) is to transform data information and knowledge into a type of visual information for intelligence officers reading, where the natural ability of mankind to identify visual mode quickly will be made most of. Information representation combines human brains with computers, which are two most powerful information processing systems. Semantic information representation and organization interface will enable us to browse and research massive data and carry out interaction with the data so that the internal features and principles hidden in the information can be found. In this society where information is getting richer day by day, Design and Implementation of Information Representation System have basically changed our way to express and interpret massive and complicated data. The effects of Information Representation System are wide and profound and can lead intelligence officers to have the new interpretation and effective decision.
\end{abstract}

\section{Introduction}

As an important assistant method for cognition ability of information, Information Representation System (IRS) is designed to assist the intelligence officers to interpret and judge information. Although the concept of "Intelligence" hasn't been confirmed yet, there are still some common agreements about the functions of it. Some scholars consider "Intelligence" as a social competition means of information and knowledge status. A part of information and a part of knowledge comprises the whole intelligence. But intelligence is not equal to the knowledge of information or information of knowledge. Intelligence is used as an important means of social competition [1].

The task of intelligence work is about collecting the new information and new knowledge related to activities of competitors by regular and irregular means, and processing the information by means of visualization sending the information to the intelligence requiring parties for final service of decision making.

As an important assistant means in intelligence forming, there are two final targets for information representation, assisting decision making support and assisting decision reference.

\section{1) Assisting decision support}

Assisting decision making is for the clear decision; process the massive data and assist decision makers to interpret the information by solution of visualization and provide a reliable decision basis for decision makers.

\section{2) Assisting decision reference}

Assisting decision reference means tracing and reporting relevant conditions about the issues and fields which the decision makers are concerned about when there is no specific decision requirement, and sending information in form of visualization to the relevant decision makers to help them interpret the information and draw their attentions. In this process, the first step is to collect relevant information and judge the truthfulness of the information; then select the valuable information and analyze and summarize the information; finally, send the information in form of visualization to the decision makers for reference.

What if the targets of assisting decision making support and assisting decision reference are completed just by words expression but not by visualization? Before answering this question, we need to know that the attentions of mankind can only last for 8 seconds (conclusion of Biological Information Research Centre of USA). With the visual system in the brain, a person can identify, store and recall the image information fast and store the concept in the image information as a longterm memory. In nowadays, visualization of data in the age of big data will improve the reading efficiency and is suitable for the biological function of human beings.

There are four main advantages lying in information representation: transforming big data into small data, strong attracting, easy to be understood and easy to be remembered. Therefore, to achieve the targets of decision support and reference, IRS will play a key part.

\section{Defining the concept of information representation}

In the age of big data, it's very hard to analyze the massive data for information; people can hardly identify the effective information and data sources. The massive data will make information interpretation harder. The old and new information will mix together, interconnect with each other and coexist. The quantity of data can be solved by tools of program development. However, the complication should also be considered; the importance can be displayed and clarified 
by means of information representation. In the 20 years' development of visualization, it has sunk into every corner of information science. The terminal target of information research is to interpret information; visualization is of a powerful ability of interpretation with "one picture beating a thousand words". The proper data visualization plan will improve the comprehension of information workers to the data.

Big data information analysis brings both challenges and chances. Information science has also evolved to studying information and knowledge and information analysis has evolved to analysis of various data. Therefore, in information analysis, information representation can be defined as "using technology methods of computer to collect and explore the various information resources in real-time way based on information analysis system, and expressing and interpreting information and hidden essence of in-formation by means of direct visual form so that the information judging efficiency can be improved and scientific decision can be assisted. In the end, reform of information analysis mode will be achieved."

In representation of information, visual forms will be used to select, transform and replace the data and visual forms in various dimensions and transform the massive data to the visual form which can be read manually to disclose relationship between different data and information and tendency to the information readers and decision makers and help the analysts to judge the current conditions and improve the level of decision.

\section{Principle of information representation system}

Can the methods of information representation play an effective role in information reading and judging and become an effective approach for information analysts to interpret and judge information? What is a scientific IRS design? Based on reflection of the questions and referring to load of cognitive load, with the research target of the program and features of information representation, the program team pointed out the following guiding principles of information representation system based on the principle of "timeliness, correctness, effectiveness and readability" (see for example Table 1): principle of scientific, understanding, explicitness, effectiveness and truthfulness.

\begin{tabular}{|l|l|l|}
\hline Principles & Explanation & Definition \\
\hline Scientific & $\begin{array}{l}\text { Ability to reflect } \\
\text { scientific knowledge } \\
\text { correctly }\end{array}$ & $\begin{array}{l}\text { Can the image reflect } \\
\text { scientific } \\
\text { correctly and truthfully? }\end{array}$ \\
\hline Understanding & $\begin{array}{l}\text { Ability to identify } \\
\text { relationships between } \\
\text { variables correctly }\end{array}$ & $\begin{array}{l}\text { Can the images maximize } \\
\text { understanding of relationship } \\
\text { between variables? }\end{array}$ \\
\hline Explicitness & $\begin{array}{l}\text { Ability to identify all } \\
\text { factors in pictures } \\
\text { visually }\end{array}$ & $\begin{array}{l}\text { Can the most important } \\
\text { factors or relationship be } \\
\text { significant in visualization? }\end{array}$ \\
\hline Effectiveness & $\begin{array}{l}\text { Ability to describe } \\
\text { complicated } \\
\text { relationship } \\
\text { simple drawing and } \\
\text { need of image and } \\
\text { image factors }\end{array}$ & $\begin{array}{l}\text { Can the images be easily } \\
\text { interpreted? Compared with } \\
\text { other methods (table, texts), } \\
\text { can image off more useful } \\
\text { form? For expressing } \\
\text { relationships, are all image } \\
\text { factors necessary? }\end{array}$ \\
\hline Truthfulness & $\begin{array}{l}\text { Ability to confirm the } \\
\text { real value of image } \\
\text { factor via size of } \\
\text { image factor relative } \\
\text { to visible and invisible } \\
\text { size }\end{array}$ & $\begin{array}{l}\text { Can image factors be } \\
\text { oriented and calibrated } \\
\text { correctly? }\end{array}$ \\
\hline
\end{tabular}

Table 1: Five principles of information visualization.

\section{Principle of scientific}

Being scientific means that information representation plan must be based on scientific theories and follow the relationship of scientific logics. Scientific thinking methods should be used for design. The displayed visualized charts should reflect scientific knowledge and logics correctly and truthfully; in terms of contents, the discussion should be sufficient and proper; in prediction conclusion, it should be scientific, timely and correct.

\section{Principle of understanding}

Principle of understanding means that the information representation charts can indicate the relationship between variables. This principle is for information representation technology and methods; when information representation technology is used to explore the objective data, the obvious and hidden factors displayed by the data should be expressed correctly in visualization charts. A solid foundation will be laid for information judging and reading only when the information hidden in the data is understood.

\section{Principle of explicitness}

Principle of explicitness means that the valuable information factors can be clearly explicitly judged in the visualization charts. Information representation is for making it easier for information analysts and decision makers to understand information and finding information. Therefore, explicitness of key factors in the visualization charts is the key point of IRS plan design; the information readers should be able to grasp the key of information and make decision promptly.

\section{Principle of effectiveness}

The principle of effectiveness means the ability to describe complicated relationship with simple methods and requirements to images and image factors. Compared with other methods (table, texts), can image off more useful form? For expressing relationships, are all image factors necessary? 
When data are described by IRS, the complicated charts may be not easy for the readers to understand the information in the data; making the information factors displayed in the charts are effective is an important principles of visualization plan.

\section{Principle of truthfulness}

Principle of truthfulness means the ability to confirm the real value of image factor via size of image factor relative to visible and invisible size and confirm the real value of the image factors. The IRS charts must express the data correctly; the correct orientation and calibration will return the most original data message and assure the correctness of information decision.

With guidance of the five principles, for IRS design, the specific information analysis should be considered; the visual representations must be combined with other representation to make an information chart easy to be read.

\section{Information representation based HWSME theory}

The information theories have been developed well with a complete system and mature methods, which are the solid foundations for the theory of information theories in the age of big data. How-ever, with the age of big data comes an opportunity to reexamine the information theories. The information must be faster and more correct because of the features of big data. The requirement of in-formation can't be met only with the knowledge and thinking of expert groups. IT must be applied to make information forming smarter.

In front of the massive data, the process ability of human brains is limited and, in theory, the process ability of computers is limitless. On the other hand, smartness equips computers with ability of simulate human sense and thinking processes and make smart computers which can identify mode and images, produce languages, play games, prove theorems automatically and design programs automatically and equip computer with experts system, learning system and smart robots, etc. however, the information is used for a specific objective and a product made by processing relevant facts, data, information, knowledge, etc. consciously. Information is the useful information processed by human brains. The basic features of information are purpose, consciousness, affiliation and labor processing. They are connected with each other and integral. Obviously, the computer can't process information. In age of big data, information work does not only need the information analysis by experts and in-formation workers but need the smarter computer technology to meet the requirement of fast real-time report. In Information work, the experts, data and developed information technology will be integrated to work out solution and provide decision wisdom. The combination of smart process of big data with experts' knowledge is complicated and can't be completed without open complex giant system and methodology.
In 1990, Mr. Qian Xuesen raised the open complex giant system and methodology, which is the Meta Synthesis from qualitative to quantitative. It developed into Hall for Workshop of Metasynthetic Engineering (HWSME) from qualitative to quantitative. This method framework is to combine expert system, machine system and knowledge system to create a smart human machine system; it created the new theory of systematic engineering. This theory is applicable in information work in age of big data.

With the guidance of Workshop of Metasynthetic Engineering (HWSME) of Qian Xuesen, the application theory of information representation is established by interpreting the physical structure of the system in the view of information (see Figure I); it consists of three parts: the first one is the experts system including the experts integrating the information; the second one is the machine system made up of the computer hardware to provide information service to information workers and experts; the third is the knowledge system including various information resources. In terms of information analysis process, the system includes three parts: the first one is the qualitative information comprehensive integration system based on judgment of experience of expert system; the second is the quantitative information analysis comprehensive integration system based on data collection and processing tools; the third is the comprehensive integration system from qualitative information to quantities information.

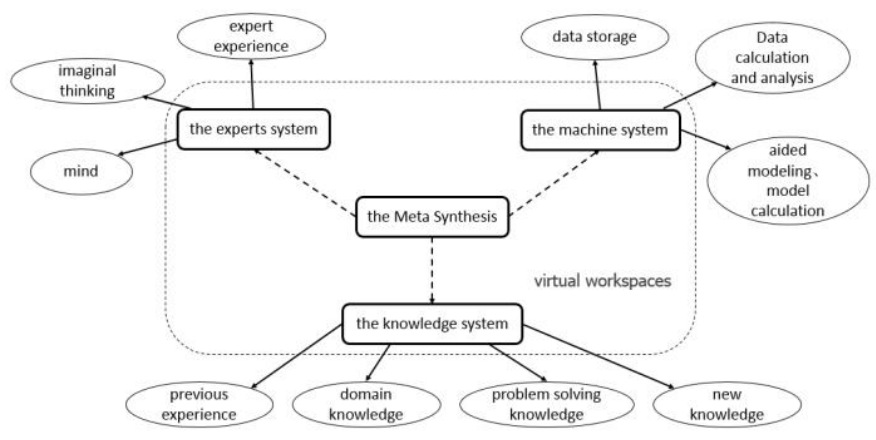

Fig. 1. Application theory framework of information representation based on Hall for WorkShop of Meta-synthetic Engineering [2]

In the age of big data, construction of IRS does not only need the expert system consisting of experts from different subjects and fields and with the proper knowledge structure necessary for facing complex giant system but to design and develop effective tools for massive data process to achieve effective collection and precise analysis of information.

Based on the theory and methods of Workshop of Metasynthetic Engineering, in the process of information forming, IRS has the three different approaches of analysis and exploration (see the figure below):

\section{A. Direct description and representation of collected data}

In this type of information representation, machine system will represent the data according to features of the data and 
information requirement via direct expression of data and provide it to expert system and complete the information production with support of knowledge system.

\section{B. Description and representation of summary of abstract data}

In this method of information representation, the abstract data is explored and analyzed by clustering, classifying, or relative data analysis in the machine system and the visualized results are displayed to expert system and the information production with support of knowledge system is completed.

\section{Description and representation of knowledge logics}

In this method of information representation, information representation of the expert knowledge plays an important role; after the experts read the information transmitted by machine system, they will judge the information according to the knowledge and display the knowledge logics visually.

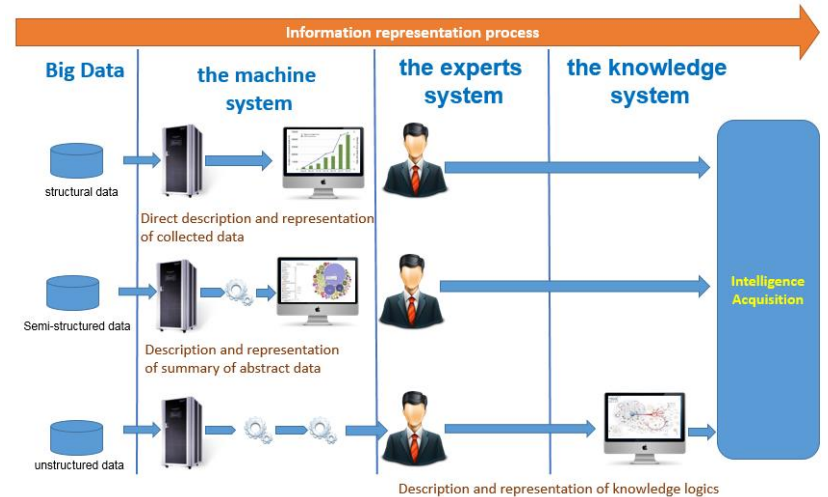

Fig.II. Information representation approach in the system of WorkShop of Metasynthetic Engineering

Workshop of Metasynthetic Engineering of man-machine intelligence is the application theory of Information Representation System and is in the category of methodology. It is the science of methods, approaches, methods and rules of information work in the age of big data. In each approach of information representation, experts can draw information decision by comparing, explaining, exploring and analyzing data and with the support of the knowledge.

\section{Development tendency and research direction of information representation system}

In the age of big data, when data flow increases exponentially, people have realized the importance to change Data Mountain into data mine. Learning the knowledge shown from the data has become an important way to intelligence acquisition. This makes the data of information exploration more complicated and various and the requirements of information representation are stricter. At present, information representation technology is also used in information analysis tools; but most of them are the simple visualization of the results.
In this article, the research background and current situations of information representation in the age of big data is analyzed, the concept of information representation has been defined, the expression classification of information representation is studied, the modules of each step built in scientific and technical information have been designed, smartness capacity of information analysis of in conditions of big data has been enhanced and the information is made more direct and effective in assisting decision.

To achieve the above targets, the article first studied the knowledge and technologies of information representation and knowledge representation and compared the cases of classic representation tools in China and abroad; then design contents and information representation application suggestions of scientific and technical information are analyzed.

As a smart information system in the age of big data, scientific and technical information representation module is designed to help decision makers to make better decisions. Therefore, the key of the system is not to check whether information representation has the advantages (appearance, luxury, etc.) but check whether the information sent to the decision makers is correct.

Research of representation of scientific and technical information is a new topic and there are few researches in this field both in China and abroad. This research is still in the early stage and many is-sues are not covered in the article and need further study.

With development of IT and requirements of information smartness, the future development tendency of information representation can be roughly seen:

1) More IRS software or platforms will appear;

2) Customized information can be pushed visually;

3) Interactive operation of information representation can be achieved, etc.

Information representation is to transform data information and knowledge into a type of visual information for reading, where the natural ability of mankind to identify visual mode quickly will be made most of. Information representation combines human brains with computers, which are two most powerful in-formation processing systems. An effective IRS interface will enable us to browse and research massive data and carry out interaction with the data so that the internal features and principles hidden in the information can be found.

In this society where information is getting richer day by day, research of IRS and application development have basically changed our way to express and interpret massive and complicated data. The effects of IRS are wide and profound and can lead us to have the new interpretation and effective decision. 


\section{References}

[1] Wang Zhijin, Lili. "Information, Knowledge-Recognition", Information Science, pp. 673-676, (2001).

[2] [2] Dai Ruwei, Li Yaodong. "Hall for WorkShop of Metasynthetic Engineering of Man and Machines", Gene China. 